\title{
François Rabelais, Gargantua e Pantagruele
}

\section{Concetta Cavallini}

\section{(2) OpenEdition}

\section{Journals}

\section{Édition électronique}

URL : https://journals.openedition.org/studifrancesi/3042

DOI : 10.4000/studifrancesi.3042

ISSN : 2427-5856

\section{Éditeur}

Rosenberg \& Sellier

\section{Édition imprimée}

Date de publication : 1 juillet 2013

Pagination : 440-441

ISSN : 0039-2944

\section{Référence électronique}

Concetta Cavallini, « François Rabelais, Gargantua e Pantagruele», Studi Francesi [En ligne], 170 (LVII I

II) | 2013, mis en ligne le 30 novembre 2015, consulté le 01 février 2023. URL : http://

journals.openedition.org/studifrancesi/3042 ; DOI : https://doi.org/10.4000/studifrancesi.3042

\section{Ce document a été généré automatiquement le 1 février 2023.}

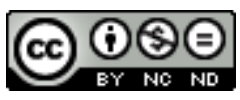

Creative Commons - Attribution - Pas d'Utilisation Commerciale - Pas de Modification 4.0 International - CC BY-NC-ND 4.0

https://creativecommons.org/licenses/by-nc-nd/4.0/ 


\title{
François Rabelais, Gargantuae Pantagruele
}

\author{
Concetta Cavallini
}

\section{RÉFÉRENCE}

FRANÇOIS RABELAIS, Gargantua e Pantagruele, introduzione e cura di Lionello sozzI.

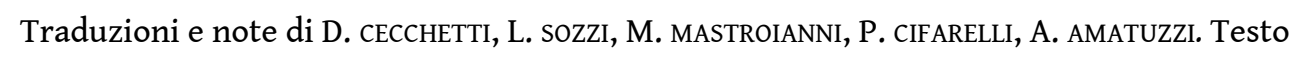

francese a fronte a cura di Mireille HuchoN, Milano, Bompiani, 2012, pp. 2146.

1 Le nouveau volume de la collection «Classici della Letteratura europea» (dirigée par Nuccio Ordine) présente la traduction de l'imposant roman de Rabelais, Gargantua et Pantagruel, en cinq volumes. Une équipe de chercheurs italiens a travaillé sous la direction de Lionello Sozzi sur le texte français établi par Mireille Huchon avec la collaboration de François Moreau en 1994 pour la Bibliothèque de la Pléiade (François Rabelais, Gargantua, Pantagruel, Tiers Livre, Quart Livre, Cinquième Livre, établissement des textes par Mme Mireille Huchon, Paris, Editions Gallimard, 1994). Dario Cecchetti s'est occupé de la traduction et des notes de Gargantua, Lionello Sozzi lui-même de Pantagruel, Michele Mastroianni du Tiers Livre, Paola Cifarelli du Quart livre et Antonella Amatuzzi du Cinquiesme livre. Lionello Sozzi, dans son introduction riche et rigoureuse (Umanesimo di Rabelais, pp. I-XLIV), situe Rabelais dans le contexte humaniste. Avec une prose fluide et élégante, il collationne les différentes opinions que la critique a avancées au cours des années sur la série des romans de Rabelais. La tâche n'est pas sans difficulté, car le profil culturel de Rabelais, ses compétences en droit, en littérature, en théologie, ses relations avec les savants de son époque, l'expérience acquise au cours de ses trois voyages en Italie aux côtés de l'évêque Jean du Bellay et de Guillaume du Bellay, gouverneur du Piémont, ont été souvent mal interprétés sur la base du style apparemment populaire et carnavalesque (selon la lecture de Bachtine) de ses romans. Sozzi examine l'incidence du thème du gigantisme, les traits concernant la dignitas, la présentation assez problématique de l'institution du mariage dans les 
romans ainsi que la position, novatrice, de Rabelais dans le domaine de l'éducation des enfants. Les renvois à l'Ecriture sainte, la présence de litanies et d'autres éléments concernant la religion sont replacés dans le domaine de la véritable attitude religieuse de l'auteur qui n'a pas encore été entièrement défriché. Les analyses de Bachtine sur le côté carnavalesque et populaire des romans de Rabelais sont reprises pour en souligner les limites, à la lumière des progrès de la critique contemporaine; la formation de Rabelais était sans aucun doute classique et son public cultivé. Voilà alors que Sozzi soutient avec force, à la fin de son introduction (xLI et suiv.) l'importance d'une relecture du langage de Rabelais pour essayer de pénétrer les raisons de sa créativité, de son exagération, de son érudition mais aussi de son incisivité populaire. Comment les choses ont-elles pu se combiner avec tant de maîtrise et des résultats si efficaces? Voici la question qui doit guider les analyses critiques. Une «Note biographique» de Rabelais (pp. XLV-XLVIII) et une «Note bibliographique» (pp. XLIX-LXI) riche et ponctuelle complètent l'introduction. Le texte français des romans reproduit le texte de base, ainsi que les variantes choisies par Mireille Huchon pour l'édition Pléiade de 1994. Pour chaque livre, dans une note critique préliminaire, les traducteurs donnent les renseignements nécessaires pour s'orienter dans l'histoire éditoriale complexe des romans de Rabelais et pour comprendre la structure de l'œuvre et le développement de l'action. Pour Gargantua, Dario CECCHETTI décrit sa composition postérieure au succès du Pantagruel en 1534 (pp. 3-5). Dans l'édition originale il manque la page de titre et donc les spécialistes se partagent entre les deux dates de 1534 ou 1535 pour sa publication chez François Juste, à Lyon. L'édition choisie comme base pour la traduction est la dernière publiée par Juste à Lyon, celle de 1542. L'histoire de Pantagruel, ainsi que le rappelle Lionello sozzi dans sa «Note critique» (pp. 389-391) est plus linéaire. L'édition choisie par Huchon et donc pour la traduction est l'édition définitive du roman publiée chez François Juste en 1542, dix ans après la première édition. Les nombreuses éditions parues entre ces deux dates compliquent la tâche des chercheurs, car il est difficile d'inférer si Rabelais collabora ou non à établir le texte pour les éditions. Le Tiers livre ouvre le "deuxième volet" de l'ensemble de Rabelais. Michele MASTRoIAnNI, sur la base des choix de Huchon, décrit les raisons de l'élection de l'édition de 1552 (Paris, Michel Fezandat) comme édition définitive: elle fut presque sûrement revue par l'auteur (pp. 721-723). Comme pour Pantagruel, le problème des contrefaçons se pose pour ce roman, sorti en 1546 et centré sur une figure nouvelle, celle de Panurge, le précepteur de Pantagruel. Du Quart livre, sorti en 1548, Rabelais publia en 1552 l'édition définitive. Un exemplaire de la deuxième édition de Michel Fezandat, publiée la même année, enrichi de corrections autographes de Rabelais, a servi de base pour l'établissement du texte français et donc pour la traduction italienne. Paola CIFARELLI explique dans sa note critique (pp. 1129-1131) que le voyage, sujet principal du Quart Livre, est une métaphore du désir de connaissance et de compréhension du réel. La note critique d'Antonella AMATUzzı sur le Cinquiesme Livre révèle toute la complexité de l'histoire éditoriale de ce texte posthume, qui va de l'établissement du texte (à partir des trois versions différentes que l'on possède) à la question de l'authenticité de cette œuvre. Mireille Huchon a choisi d'utiliser le texte de l'édition de 1564, publiée après la première, fortement incomplète, de 1562. Cette édition est redevable, avec tout ce que cette affirmation comporte, au travail de l'éditeur inconnu qui s'est occupé d'établir le texte pour la publication. Si la critique semble désormais unanime quant à l'authenticité du livre, elle est encore partagée sur le sens et sur le rôle qu'il faut lui donner à l'intérieur de l'ensemble des cinq romans de Rabelais. La traduction italienne, qui arrive après la 
traduction magistrale d'Augusto Frassineti (Sansoni, 1980) et après celle de Mario Bonfantini (publiée chez Einaudi et chez Rizzoli), est fluide et rigoureuse et reproduit de manière assez fidèle le rythme, le souffle, l'agencement du texte de départ, ce qui est méritoire. Les notes sont placées cumulativement à la fin de l'ensemble des traductions, choix qui a l'avantage de ne pas interrompre la lecture du texte de Rabelais. Elles donnent des renseignements linguistiques, culturels, mythologiques et littéraires, et éclaircissent pour le lecteur les renvois internes aux romans, tels ceux sur les thèmes et personnages récurrents. L'apparat critique est ponctuel tout en étant mesuré, et n'alourdit pas la traduction. L'index «dei personaggi e delle cose notevoli nel testo» (pp. 2077-2104) et l'index des noms de l'introduction et des notes (pp. 2105-2127), ainsi que la table des correspondances entre les noms français et les noms italiens (pp. 2129-2146) ont été réalisés par l'équipe éditoriale. Un bref profil biographique des cinq traducteurs et éditeurs des romans de Rabelais est placé en clôture. La communauté scientifique italienne ainsi que les lecteurs et les amateurs de Rabelais savent gré à Lionello Sozzi et à ses collègues pour cette nouvelle traduction, qui aligne le texte de Rabelais sur les nouvelles découvertes et sur les dernières hypothèses formulées pas la critique, tout en reproposant dans le texte italien la magie et la fascination invariées d'une langue qui demeure encore, après quatre siècles, inimitable et sans égale. 